Z. klin. Chem. u. klin. Biochem.

8. Jg., S. 221-224, Mai 1970

\title{
Gaschromatographische Bestimmung von Testosteron im Urin ${ }^{1}$ )
}

\author{
Von H.-Ch. Curtius, M. Zachmann und M. Müller \\ Aus der Medizinisch-Chemischen Abteilung der Universitäts-Kinderklinik Zïrich (Direktor: Prof. Dr. A. Prader)
}

(Eingegangen am 11. November 1969)

Es wird eine gaschromatographische Methode zur Bestimmung von Testosteronglucuronid im Urin beschrieben.

Die Methode umfaßt Hydrolyse mit $\beta$-Glucuronidase, Extraktion mit Äther, Vorreinigung mit Kolonnen- und Dünnschichtchromatographie, Silylierung und Gaschromatographie. Die Verluste während der Vorreinigung werden durch Zugabe von [ $\left.{ }^{3} \mathrm{H}\right]$-Testosteron als interner Standard kompensiert.

Die Werte gesunder Versuchspersonen und Patienten mit endokrinen Störungen sind tabellarisch zusammengefaßt.

\section{The Gas chromatographic determination of urinary testosterone}

A method for the determination of testosterone glucuronide in urine using gas-liquid chromatography is described. The method consists of hydrolysis with $\beta$-glucuronidase, extraction with ether and prepurification on an alumina column and thin-layer chromatography. Gas-liquid chromatography of the trimethylsilyl ether derivative is then performed.

Losses during prepurification are compensated by adding $\left[{ }^{3} \mathrm{H}\right]$-testosterone as internal standard. Examples of values of normal subjects and of patients with endocrine disorders are presented.

Seit Beginn der sechziger Jahre wurden mehrere Methoden zur Bestimmung von Testosteron im Urin veröffentlicht (1-20). Unter den kolorimetrischen Methoden hat sich diejenige von CAMACHO und Mrgeon (2) bewährt. Zur Erfassung geringer Testosteronmengen, wie sie bei pathologischen Zuständen und vor der Pubertät auftreten, ist ihre Empfindlichkeit jedoch zu gering. Eine fluorimetrische Methode wurde von GraEF und Mitarbeitern (15) beschrieben. Versuche mit der Proteinbindungsmethode sind zwar erfolgversprechend für die Praxis, aber bis heute noch zu unspezifisch und in niederen Konzentrationsbereichen ungenau (21). Die Methode von SANDBERG und Mitarbeitern (7), die auf der gaschromatographischen Bestimmung nach Hydrolyse und Vorreinigung nach der Methode von Caмacho und Mrgeon (2) beruht, hat sich als zuverlässig erwiesen (22). Die Technik der Gradienten-Elution gestaltet dieses Verfahren jedoch relativ zeitraubend. Auch die gaschromatographische Methode von WATSON (23) bedient sich der Gradienten-Elution.

Ziel der vorliegenden Arbeit ist es, eine spezifische und empfindliche Methode zur gaschromatographischen Bestimmung von Testosteron im Urin zu beschreiben, die besonders zur Bestimmung geringer Konzentrationen geeignet und relativ wenig aufwendig ist.

\section{Methodik}

\section{Geräte}

Gaschromatograph „F 20 “ der Fa.'Perkin-Elmer mit Flammenionisationsdetektor.

GC/MS Kombination LKB ,9000“.

Liquid Scintillation Spektrometer Modell „3324 Tri-Carb“ der Fa. Packard.

UV-Lampe 253 nm.

1) Mit Unterstützung des Schweiz. Nationalfonds zur Förderung der wissenschaftlichen Forschung, Kredit Nr. 36868.
Chromatographierohr, verjüngt von $350 \times 15 \mathrm{~mm}$ auf $140 \times 8 \mathrm{~mm}$, mit Hahn, 3 mm Bohrung.

\section{Chemikalien}

Aceton p. a., Merck, Art.-Nr. 14

Âthylalkohol für UV-Spektroskopie, Fluka, Art.-Nr. 02850

Aluminiumoxid $\mathrm{Al}_{2} \mathrm{O}_{3}$, Woelm neutral, Aktivitätsstufe $\mathrm{I}$, Woelm, Eschwege

Benzol purum, thiophenfrei, Ph. Helv. I, Fluka, Art.-Nr. 12560 (destillieren)

Chloroform $\mathrm{CHCl}_{3}$ p. a., Merck, Art.-Nr. 2445

Diäthyläther puriss p. a., Fluka, Art.-Nr. 31690

Essigsäureäthylester p. a., Merck, Art.-Nr. 9623 (destillieren)

$\beta$-Glucuronidase (bakteriell) Typ I, Sigma, Art.-Nr. 105-8

Hexamethyldisilazan purum, Fluka, Art.-Nr. 52620

Kaliumdihydrogenphosphat $\mathrm{KH}_{2} \mathrm{PO}_{4}$, Merck, Art.-Nr. 4873

Kieselgel HF 254 nm nach StahL, Merck, Art.-Nr. 7739

Natriumbydroxid $\mathrm{NaOH}$ p. a., Merck, Art.-Nr. 6498

Natriumsulfat $\mathrm{Na}_{2} \mathrm{SO}_{4}$ wasserfrei p. a., Merck, Art.-Nr. 6649

Quarzsand $0,1-0,3 \mathrm{~mm} \varnothing$

Salzsäure $32 \% \mathrm{HCl}$ p. a., Merck, Art.-Nr. 319

Stigmasterin, Fluka, Art.-Nr. 57399

Testosteron für biochem. Zwecke, Merck, Art.-Nr. 8973

Testosteron-[1, 2- $\left.{ }^{3} \mathrm{H}\right]$ 0,2 mC, $15000 \mathrm{mC} / \mathrm{mMol}$, TRC, Amersham

Trimethylchlorsilan puriss, Fluka, Art.-Nr. 92360

POPOP $=1,4-D i\left(5^{\prime}\right.$-phenyl-oxazol-2'-yl)benzol, Packard

$\mathrm{PPO}=2,5$-Diphenyloxazol, Packard

\section{Reagenzien}

Phosphatpuffer: $\mathrm{pH} 6,2 ; 0,5 \mathrm{M} ; 60 \mathrm{~g} \mathrm{KH}_{2} \mathrm{PO}_{4}$ in $500 \mathrm{ml} \mathrm{H} \mathrm{H}_{2} \mathrm{O}$ lösen, mit $2 \mathrm{~N} \mathrm{NaOH}$ auf $\mathrm{pH} 6,2$ einstellen, auf $1 l$ auffüllen mit $\mathrm{H}_{2} \mathrm{O}$.

Natronlange: etwa $1 \mathrm{~N} ; 40 \mathrm{~g} \mathrm{NaOH}$ werden in $1 \mathrm{l}$ Wasser gelöst.

Aluminiumoxid desaktiviert: $0,6 \mathrm{~m} l \mathrm{H}_{2} \mathrm{O}$ mit $10 \mathrm{~g} \mathrm{Al}_{2} \mathrm{O}_{3}$ während $10 \mathrm{Min}$. schütteln. Erst nach einstïndigem Stehenlassen verwenden. Elutionsmittel Säulencbromatograpbie: $0,1 \%$ Athanol in Benzol; $0,25 \%$ Athanol in Benzol.

Lösungen für Aktivitätszäblung: Lösung I: $5 \mathrm{~g}$ PPO, $300 \mathrm{mg}$ POPOP in 1 l Toluol; Lösung II: $3,5 \mathrm{~g}$ PPO, $150 \mathrm{mg}$ POPOP, $50 \mathrm{~g}$ Naphthalin in $500 \mathrm{~m} l$ Dioxan.

Lösung $\left[{ }^{3} \mathrm{H}\right]-$ Teslosteron in Athanol: $75000 \mathrm{Imp} . / \mathrm{Min} .\left[{ }^{3} \mathrm{H}\right]-$ Testosteron/1 ml Äthanol. 
Stigmasterinlösung: $4 \mathrm{mg}$ Stigmasterin in $100 \mathrm{ml}$ Athanol $(100 \mu l$ $=4 \mu \mathrm{g}$ ).

Testosteronlösung: $5 \mathrm{mg}$ Testosteron in $100 \mathrm{ml}$ Åthanol.

Testosteron-Eichkurve: (bei jeder Gaschromatographieserie)

$10 \mu \mathrm{g}$ Testosteron

$5 \mu \mathrm{g}$ Testosteron

$2 \mu \mathrm{g}$ Testosteron

$0,5 \mu \mathrm{g}$ Testosteron

Arbeitsvorschrift

Hydrolyse mit $\beta$-Glucuronidase

Einzusetzende Urinmenge:

$\begin{array}{ll}\text { Männer } & 1 / 5 \operatorname{der} 24 \text { Stdn.-Menge } \\ \text { Frauen } & 1 / 3 \operatorname{der} 24 \text { Stdn.-Menge } \\ \text { Kinder (bis Pubertät) } & 3 / 5 \operatorname{der} 24 \text { Stdn.-Menge }\end{array}$

Die jeweilige Urinmenge wird durch Zugabe von Phosphatpuffer auf $\mathrm{pH} 6,2$ gebracht ( $1 / 5$ des Urinvolumens). Anschließend werden 100 Einheiten $\beta$-Glucuronidase $/ \mathrm{m} l$ Urin zugegeben. Das Gemisch wird $72 \mathrm{Stdn}$. bei $37^{\circ}$ im Wärmeschrank inkubiert, zur Verhinderung von bakterieller Zersetzung werden 3 Tropfen Chloroform zugefügt. Anschließend gibt man $0,2 \mathrm{ml}\left[{ }^{3} \mathrm{H}\right]$-Testosteron zu. Von $0,2 \mathrm{ml}\left[{ }^{3} \mathrm{H}\right]$-Testosteron wird der $100 \%$-Wert im „liquid scintillation counter" ermittelt.

\section{Extraktion}

Das Hydrolysat wird $3 \mathrm{mal}$ mit gleichen Volumenteilen Äther extrahiert. Die vereinigten Ätherextrakte werden anschließend $3 \mathrm{mal}$ mit 0,1 Volumenteilen $1 \mathrm{~N} \mathrm{NaOH}$ ausgeschüttelt und mit $\mathrm{H}_{2} \mathrm{O}$ neutral gewaschen. Der Äther wird mit Natriumsulfat getrocknet und am Vakuum eingedampft. Restliches Wasser wird mit Äthylalkohol azeotrop abdestilliert.

\section{Aluminiumoxid-Chromatograpbie}

$4 \mathrm{~g}$ desaktiviertes Aluminiumoxid werden in Benzol auf die Kolonne gegeben. Der getrocknete Ätherextrakt wird in $5 \mathrm{~m} l$ Benzol gelöst und auf die Kolonne überführt. Nach einmaligem Waschen mit $5 \mathrm{~m} l$ Benzol-Äthanol $(99,9: 0,1)$ chromatographiert man zunächst mit $10 \mathrm{ml}$ Benzol-Äthanol $(99,9: 0,1 \mathrm{v} / \mathrm{v}$ ) (= Fraktion 1a) und anschließend nochmals mit $10 \mathrm{~m} l$ Benzol-Äthanol $(99,9: 0,1 \mathrm{v} / \mathrm{v})$ (= Fraktion $1 \mathrm{~b})$. Daraufhin wird zunächst mit $30 \mathrm{~m} l$ Benzol-Äthanol $(99,75: 0,25 \mathrm{v} / \mathrm{v})(=$ Fraktion $2 \mathrm{a})$ und dann mit $15 \mathrm{~m} l$ dieser Mischung (= Fraktion $2 \mathrm{~b}$ ) und zuletzt nochmals mit $15 \mathrm{ml}$ (= Fraktion 2c) chromatographiert. Alle Fraktionen werden in Meßzylindern aufgenommen, gemischt und $1 / 20$ des jeweiligen Volumens zur Aktivitätsmessung in den „liquid scintillation counter" gegeben. Bei zu geringer Gesamtaktivität werden eventuell weitere Fraktionen a $20 \mathrm{~m} l$ mit Benzol-Äthanol $(99,75: 0,25 \mathrm{v} / \mathrm{v})$ chromatographiert. Die Fraktionen $1 \mathrm{a}$ und $1 \mathrm{~b}$ sollten kein $\left[{ }^{3} \mathrm{H}\right]-$ Testosteron enthalten. In Fraktion $2 \mathrm{a}$ findet sich der Hauptanteil und eventuell in $2 \mathrm{~b}$ noch eine geringere Menge. Diese aktiven Fraktionen werden vereinigt und eingedampft. Der Rückstand wird $3 \mathrm{mal} \mathrm{mit} 2 \mathrm{ml}$ Aceton in ein kleines Schliffröhrchen überführt und das Lösungsmittel vorsichtig im Stickstoffstrom abgeblasen.

\section{Messung der Radioaktivität}

Der jeweilige Rückstand wird im Scintillatorfläschchen mit $0,2 \mathrm{ml}$ 96proz. Äthanol, $5 \mathrm{~m} l$ Scintillatorflüssigkeit I und $0,5 \mathrm{~m} l$ Scintillatorflüssigkeit II versetzt und die jeweilige Radioaktivität gemessen.

\section{Dünnschichtchromatograpbie}

Das trockene Eluat wird in $100 \mu l$ Aceton gelöst und in einer $5 \mathrm{~cm}$ breiten Zone auf eine Dünnschichtplatte aufgetragen. Nach zweimaligem Nachspülen mit $100 \mu l$ Aceton trägt man auf beiden Seiten der Platte je $10 \mu \mathrm{g}$ Testosteron auf und entwickelt im
System $\mathrm{CHCl}_{3}$ - thylacetat $(2: 1 \mathrm{v} / \mathrm{v})$ bei $4^{\circ}$. Das Fließmittel werden $15 \mathrm{~cm}$ hoch laufen gelassen. Die Platte wird anschließend unter . der UV-Lampe bei $253 \mathrm{~nm}$ betrachtet und eine $16 \mathrm{~mm}$ breite Zone auf der Höhe des Testosterons markiert, das Kieselgel abgekratzt und in eine kleine Chromatographiesäule über $200 \mathrm{mg}$ Quarzsand und Glaswolle eingefüllt und mit $5 \mathrm{~m} /$ Äther eluiert.

Vorbereitung der Proben für die Gaschromatograpbie und Radioaktivitätsmessung

Das getrocknete Eluat wird in $10 \mathrm{ml}$ Aceton gelöst. Davon werden $1 \mathrm{~m} l$ für die Messung der Radioaktivität, $4 \mathrm{~m} l$ für die Gaschromatographie ohne internen Standard und $4 \mathrm{ml}$ für die Gaschromatographie mit internem Standard ( $4 \mu \mathrm{g}$ Stigmasterin) verwendet. Das Lösungsmittel in den einzelnen Gläschen wird vorsichtig unter Stickstoff abgeblasen. Die Proben für die Gaschromatographie werden etwa 10 Min. im Exsikkator über $\mathrm{P}_{2} \mathrm{O}_{5}$ getrocknet.

\section{Silylierung}

Die Proben für die Gaschromatographie werden in $100 \mu l$ Silylierungsgemisch gelöst (Ubbetstand verwenden) und $1 \mathrm{Std}$. bei $60^{\circ}$ inkubiert. Das Silylierungsgemisch wird im trockenen Stickstoffstrom abgeblasen und der Trockentückstand erneut in $20 \mu \mathrm{l}$ Silylierungsgemisch gelöst.

\section{Gaschromatograpbie}

Zur Gaschromatographie verwenden wir einen Perkin-Elmer Fraktometer (Modell „F 20“) mit Flammenionisationsdetektor.

\section{Trennsäulenbedingungen}

$\mathrm{XE} 60,3 \%, 2 \mathrm{~m}$, auf Gaschrom P (100-120 mesh); $\mathrm{T}_{\mathrm{c}}=215^{\circ}$, $\mathrm{T}_{\mathrm{J}}=270^{\circ} ; \mathrm{N}_{2}=45 \mathrm{~m} / / \mathrm{Min}$.

\section{Auswertung und Berechnung}

Zur Berechnung verwendeten wir eine graphische Eichkurve (24). Anhand von Eichlösungen mit konstantem internem Standard (Stigmasterin), mit denen der ganze Analysengang durchgeführt wird, errechnet man einen Faktor (f):

$$
f=\frac{F_{x}}{F_{S t}}=\frac{\text { (Fläche Testosteron) }}{\text { (Fläche Stigmasterin) }}
$$

(Fläche $=$ Peakhöhe $\times$ Peakbreite in der halben Höhe)

Auf der Abszisse trägt man die eingesetzte Menge, auf der Ordinate den errechneten Faktor $f$ ab. In einem Chromatogramm mit unbekannten Mengen bestimmt man den Faktor $f$ und liest auf der Eichkurve ab.

\section{Resultate}

Abbildung 1a zeigt das Gaschromatogramm eines Testgemisches und $1 \mathrm{~b}$ das Gaschromatogramm eines Urinextraktes.

Einige Resultate sind in Tabelle 1 (gesunde Versuchspersonen) und Tabelle 2 (Patienten mit endokrinen Störungen) zusammengefaßt.

\section{Tab. 1}

Ausscheidung von Testosteronglucuronid bei gesunden Versuchspersonen

\begin{tabular}{cccc}
\hline & $\overline{\mathbf{x}}$ & Bereich & $\mathrm{n}$ \\
\hline & & & \\
\hline Knaben & & & \\
0-4 Jahre & 0,9 & $<0,5-1,7$ & 4 \\
4-12 Jahre & 1,4 & $<0,5-3,3$ & 6 \\
12-18 Jahre & 10,8 & $2,2-27,6$ & 5 \\
Männer & & $32,5-134,5$ & 9 \\
18-38 Jahre & 71,7 & & \\
Frauen ‥ & & $7,3-12,7$ & 3 \\
27-38 Jahre & 9,4 &
\end{tabular}


Tab. 2

Ausscheidung von Testosteronglucuronid bei Patienten mit endokrinen Störungen

\begin{tabular}{|c|c|c|c|}
\hline $\begin{array}{l}\text { Alter in } \\
\text { Jahren }\end{array}$ & Geschlecht & Diagnose & $\begin{array}{l}\text { Testosteron } \\
\mu \mathrm{g} / 24 \text { Stdn. }\end{array}$ \\
\hline \multicolumn{4}{|c|}{ Erhöhte Werte } \\
\hline 5 & $\sigma^{\prime}$ & Idiopathische Pubertas praecox & 38,8 \\
\hline 16 & 우 & $\begin{array}{l}\text { Adrenogenitales Syndrom } \\
\text { (21-Hydroxylasedefekt) unbehandelt }\end{array}$ & 135,0 \\
\hline 29 & 우 & Hirsutismus & 26,3 \\
\hline 38 & q & $\begin{array}{l}\text { Virilisierendes Nebennieren- } \\
\text { rindenadenom }\end{array}$ & $\begin{array}{l}28,2 \\
47,6\end{array}$ \\
\hline \multicolumn{4}{|c|}{ Erniedrigte Werte } \\
\hline 9 & $\sigma^{\prime}$ & Anorchie & $<0,5$ \\
\hline 17 & $0^{\circ}$ & $\begin{array}{l}\text { Hodenatrophie nach doppel- } \\
\text { seitiger Hodentorsion }\end{array}$ & 4,4 \\
\hline 18 & $\sigma^{*}$ & Pubertas tarda & 2,0 \\
\hline 18 & $0^{\prime}$ & Pubertas tarda & 2,1 \\
\hline
\end{tabular}

Tab. 3

Reproduzierbarkeit der Methode. Resultate von 5 Bestimmungen von Testosteronglucuronid im gleichen Urin eines Patienten mit adrenogenitalem Syndrom

\begin{tabular}{|c|c|}
\hline \multirow{5}{*}{\multicolumn{2}{|c|}{$\begin{array}{l}\text { 1. } 154,5 \mu \mathrm{g} / 24 \text { Stdn. } \\
\text { 2. } 154,0 \mu \mathrm{g} / 24 \text { Stdn. } \\
\text { 3. } 152,0 \mu \mathrm{g} / 24 \text { Stdn. } \\
\text { 4. } 147,0 \mu \mathrm{g} / 24 \text { Stdn. } \\
\text { 5. } 164,0 \mu \mathrm{g} / 24 \text { Stdn. }\end{array}$}} \\
\hline & \\
\hline & \\
\hline & \\
\hline & \\
\hline Mittelwert: & $\bar{x}=154,3 \mu \mathrm{g} / 24 \mathrm{Stdn}$ \\
\hline \multirow{2}{*}{\multicolumn{2}{|c|}{$\begin{array}{ll}\text { Standardabweichung: } & s= \pm 5,53 \\
\text { Standardfehler des Mittels: } & \sigma= \pm 2,47\end{array}$}} \\
\hline & \\
\hline \multicolumn{2}{|c|}{ Variationskoeffizient: $\quad \mathrm{V}=3,6 \%$} \\
\hline
\end{tabular}

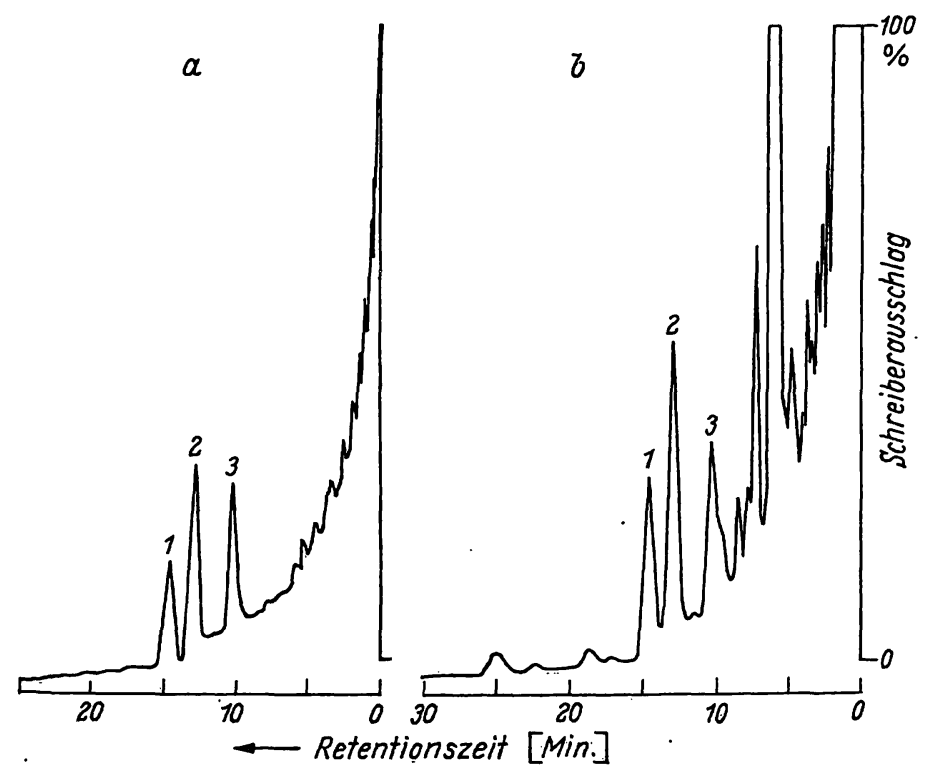

Abb. 1 a

Gaschromatogramm eines Testgemisches mit Stigmasterin als internem Standard (Trennbedingungen siehe Text).

$$
\begin{aligned}
& 1=\text { Stigmasterin } \\
& 2=\text { Testosteron } \\
& 3=\text { Epitestosteron }
\end{aligned}
$$

\section{Arbeitsaufizand}

Eine geübte Laborantin kann pro Woche 5 Bestimmungen durchführen.

\section{Spezifität und Empfindlicbkeit}

Die Spezifität wurde massenspektrometrisch überprüft. Die Nachweisgrenze liegt bei 0,5 $\mu \mathrm{g} / 24 \mathrm{Stdn}$.-Urin.

\section{Reproduzierbarkeit und Wiederauffindungsrate}

Die durchschnittliche Ausbeute von 30 Bestimmungen beträgt $81,8 \%$. Standardabweichung und Variationskoeffizient $(n=5)$ sind in Tabelle 3 wiedergegeben.

\section{Diskussion}

In der vorliegenden Arbeit wird eine spezifische, empfindliche und für Routineanalysen geeignete Methode zur Bestimmung von Testosteronglucuronid im Urin beschrieben, die sich in unserer Klinik seit längerer Zeit bewährt hat. Sie eignet sich nicht nur zum Nachweis größerer Testosteronmengen bei Erwachsenen und bei endokrinen Störungen mit Virilisierung, sondern auch zur Erfassung von geringen Testosteronmengen (bis $0,5 \mu \mathrm{g} / 24 \mathrm{Stdn}$.), wie sie bei Patienten vor und zu Beginn der Pubertät gefunden werden. Sowohl Epitestosteron wie auch das zur Medikation verwendete Methyltestosteron interferieren bei der vorliegenden Methode nicht.

Das Interesse der pädiatrischen Endokrinologie an einer möglichst frühzeitigen Erfassung von Gonadenfunktionsstörungen hat zugenommen, seitdem bekannt ist, $\mathrm{da} B$ die Hypothalamus-Hypophysen-Gonaden-Achse schon vor der Pubertät funktionstüchtig ist und daß auch präpubertale Kinder geringe Mengen Testosteron ausscheiden.

Die älteren kolorimetrischen und fluorimetrischen Bestimmungsmethoden genügen den Anforderungen nicht mehr, weil sie zu wenig spezifisch (keine Trennung von in gleicher Konzentration vorhandenem, aber diagnostisch weniger wichtigem Epitestosteron) oder zu wenig empfindlich sind. Neuere gaschromatographische Methoden, insbesondere diejenige von SANDBERG und Mitarbeitern (7) sind zwar genügend spezifisch und empfindlich, verlangen jedoch wegen der notwendigen Gradienten-Elution einen relativ großen Arbeitsaufwand.

Es ist bekannt, daß das im Urin ausgeschiedene Testosteron nicht nur aus den Gonaden stammt, sondern auch peripher aus anderen Steroiden, insbesondere aus Dehydroepiandrosteron und $\Delta 4$-Androstendion, entstehen kann. Trotzdem ist es von diagnostischem Wert, Testosteron im Urin zu bestimmen, da die Testosteronausscheidung mit der Produktionsrate von Testosteron korreliert (25) und da die bestehenden Methoden zur Bestimmung von Testosteron im Plasma teils mit praktischen (gaschromatographische Methoden: relativ große Plasmamengen erforderlich), teils mit technischen (Proteinbindungsmethoden: geringe Spezifität) Schwierigkeiten verbunden sind.

Wir danken Frau C. Trebo für ihre technische Mitarbeit. 


\section{Literatur}

1. SChUBERT, K. und K. WeHRBERGER, Naturwissenschaften 47, 281 (1960). - 2. Camacho, A. M. und C. J. Migeon, J. Clin. Endoct. Springfield 23, 301 (1963). - 3. FutreRwerr, W., N. L. McNiven, I. Narcus, C. Lantos, M. Drowdowsky und R. I. Dorfman, Steroids 1, 628 (1963). - 4. Vermeulen, A. und J. C. M. Verplancke, Steroids 2, 543 (1963). - 5. Brooks, R. V., Steroids 4, 117 (1964). - 6. Ibayashi, H., M. Nakamura, S. MuraKawa, T. Uchrkawa, T. Tanioka und K. Nakao, Steroids 3, 559 (1964). - 7. Sandberg, D. H., N. Ahmad, W. W. Clevveland und K. Savard, Steroids 4, 557 (1964). - 8. Vorgt, K. D., U. Volkwern und J. TAMM, Klin. Wschr. 42, 642 (1964). 9. Lim, N. Y. und J. F. Dingman, J. Clin. Endocr., Springfield 25, 563 (1965). - 10. Panicuccr, F., Androgens, Proc. 2nd Symposium on Steroid Hormones Ghent (1965). - 11. RosNER, J. M., N. F. Conte, J. H. Briggs, P. Y. Chao, E. M. Sudman und P. H. Forsham, J. Clin. Endocr., Springfield 25, 95 (1965). 12. vaN der Molen, H. J., D. GRoen, J. H. vaN der MaAs, Steroids 6, 195 (1965). - 13. ZURBrüGG, R. P., R. D. B. JaCOBS und L. I. Gardner, J. Clin. Endocr., Springfield 25, 315 (1965).
14. Gupta, D. und J. Goodwin, Steroids 8, 195 (1966). 15. Graef, V., P. Jobst und HJ. Staudinger, diese $Z .6,159$ (1968). - 16. IsmaIL, A. A. A. und R. A. Harkness, Biochem. J. 99, 717 (1966). - 17. MCRoberis, J. W., A. D. Olson und W. L. HerrmanN, Clin. Chem. (New York) 14, 565 (1968). 18. Schollberg, K., E. SeIler und G. K. Hinkel, Zschr. Kinderhk. 102, 341 (1968). - 19. StAJB, W. und W. HüBNER, in (Tamm, J., Ed.) Testosteron, Proc. Workshop Conference, Tremsbüttel (1967), Georg Thieme Verlag, Stuttgart (1968). - 20. MouGEy, E. H., D. R. Coltins, R. M. Rose und J. W. Mason, Analytic. Biochem. 27, 343 (1969). - 21. RuDd, B. T., R. L. RoseNFIeld, A. M. BonglovanNi und W. R. Ererlein, Steroids 13, 227 (1969). - 22. ZachmanN, M., W. W. Cleveland, D. H. SaNDBerg und W. L. Nyhan, Amer. J. Dis. Child. 112, 283 (1966). 23. Watson, J. T., J. Chromatog. 43, 339 (1969). - 24. Curtrus, H.-Ch., diese Z. 4, 114 (1966). - 25. Vermeulen, A., in (Tamm, J., Ed.) Testosteron, Proc. Workshop Conference, Tremsbüttel (1967), Georg Thieme Verlag, Stuttgart (1968).

P. D. Dr. H.-Ch. Curtius $\mathrm{CH} 8032$ Zürich Steinwiesstr. 75 